Research Article

\title{
Ginsenoside Rg1 Alleviates Podocyte Injury Induced by Hyperlipidemia via Targeting the mTOR/NF- $\kappa$ B/NLRP3 Axis
}

\author{
Tao Wang $\mathbb{D},{ }^{1,2}$ Yanbin Gao $\mathbb{D}^{1,},{ }^{1,3}$ Rongchuan Yue $\mathbb{D},{ }^{4}$ Xiaolei Wang, ${ }^{1}$ Yimin Shi, ${ }^{1}$ Jiayi Xu, ${ }^{1}$ \\ Bingjie $\mathrm{Wu},{ }^{1}$ and Yimeng $\mathrm{Li}^{1}$ \\ ${ }^{1}$ School of Traditional Chinese Medicine, Capital Medical University, 10 Youanmenwai, Xitoutiao, Fengtai District, \\ Beijing, China \\ ${ }^{2}$ School of North Sichuan Medical College, Nanchong 637000, China \\ ${ }^{3}$ Beijing Key Lab of TCM Collateral Disease Theory Research, 10 Youanmenwai, Xitoutiao, Fengtai District, Beijing, China \\ ${ }^{4}$ Department of Cardiology, Affiliated Hospital of North Sichuan Medical College, Nanchong 637000, China
}

Correspondence should be addressed to Yanbin Gao; dfyynfm@163.com and Rongchuan Yue; 251864693@qq.com

Received 26 April 2020; Revised 3 August 2020; Accepted 27 September 2020; Published 9 October 2020

Academic Editor: Tung Nguyen

Copyright ( $) 2020$ Tao Wang et al. This is an open access article distributed under the Creative Commons Attribution License, which permits unrestricted use, distribution, and reproduction in any medium, provided the original work is properly cited.

\begin{abstract}
Background. Podocyte injury plays an important role in diabetic nephropathy (DN). The aim of this study was to determine the potential therapeutic effects of the ginsenoside $\operatorname{Rg} 1$ on hyperlipidemia-stressed podocytes and elucidate the underlying mechanisms. Methods. In vitro and in vivo models of $\mathrm{DN}$ were established as previously described, and the expression levels of relevant markers were analyzed by Western blotting, real-time Polymerase Chain Reaction (PCR), immunofluorescence, and immunohistochemistry. Results. Ginsenoside $\operatorname{Rg} 1$ alleviated pyroptosis in podocytes cultured under hyperlipidemic conditions, as well as in the renal tissues of diabetic rats, and downregulated the mammalian target of rapamycin (mTOR)/NF- $\kappa \mathrm{B}$ pathway. In addition, Rg1 also inhibited hyperlipidemia-induced NLRP3 inflammasome in the podocytes, which was abrogated by the mTOR activator L-leucine (LEU). The antipyroptotic effects of Rg1 manifested as improved renal function in the DN rats. Conclusion. Ginsenoside Rg1 protects podocytes from hyperlipidemia-induced damage by inhibiting pyroptosis through the mTOR/NF- $\kappa \mathrm{B} /$ NLRP3 axis, indicating a potential therapeutic function in DN.
\end{abstract}

\section{Introduction}

Diabetic nephropathy (DN) is a progressive microvascular complication of diabetes mellitus (DM), and the primary cause of end-stage renal diseases (ESRD) [1]. Although the exact mechanism is unclear, several studies have implicated podocyte injury as the key etiological factor [2-4]. Podocytes are specialized glomerular epithelial cells that form the outermost filtration layer, and their loss/damage is a hallmark of various reno-pathological conditions including DN $[5,6]$. Pyroptosis, a type of programmed cell death characterized by swelling, pore formation, and release of proinflammatory cytokines (interleukin- (IL-) $1 \beta$ and IL-18), is a potential mechanism of podocyte loss. In fact, pyroptosis has been observed during the development of acute kidney disease and $\mathrm{DN}$, although the specific mechanism is unknown [7].
The pyroptotic cascade is triggered by the nucleotide oligomerization domain- (NOD-) like receptor family pyrin domain containing 3 (NLRP3) inflammasome, a multiprotein complex consisting of NLRP3, apoptosis-associated speck-like protein (ASC) with caspase recruitment domain (CARD) domain and procaspase-1 $[8,9]$, and the downstream inflammatory pathway is driven by caspase- 1 $[10,11]$. NLRP3 is activated by mTOR, a highly conserved serine/threonine kinase (Ser/Thr) that regulates cell growth and proliferation [12], in different in vivo and in vitro models $[13,14]$. Recent studies show that high glucose and fat levels can activate NLRP 3 inflammasomes and caspase-1 triggered pyroptosis $[15,16]$. The inflammatory mTOR-NLRP3-IL- $1 \beta$ axis is activated during lung injury, and its inhibition can reduce the immunopathological damage in lung tissues [17]. The nuclear factor kappa-B (NF- $\kappa \mathrm{B})$ and mTOR signaling 
pathways are highly interrelated $[18,19]$. While overexpression of mTOR activates NF- $\kappa \mathrm{B}$, inhibition of mTOR phosphorylation by rapamycin blocks the AKT/NF- $\kappa \mathrm{B}$ pathway [20]. In a previous study, we found that inhibiting NF- $\kappa \mathrm{B}$ can protect podocytes and reverse $\mathrm{DN}$ by blocking mesangial transdermal differentiation [21]. Therefore, we hypothesized that the mTOR-NLRP3-IL-1 $\beta$ axis is a potential target for inhibiting podocyte injury in DN.

Ginseng has been used in traditional Chinese medicine formulations for over 2000 years, and has antitransdifferentiation, antioxidation, antiapoptosis, and proautophagy effects [22-24]. The pharmacological effects of ginseng are mainly attributed to the ginsenoside Rg1, which can effectively inhibit apoptosis and protect podocytes $[25,26]$. Rg1 also induces autophagy and inhibits apoptosis by activating the $\mathrm{AMPK} / \mathrm{mTOR}$ pathway [27]. In this study, we analyzed the role of pyroptosis in DN and the therapeutic effects of Rg1.

\section{Materials and Methods}

2.1. Main Reagents. Ginsenoside Rg1 (Figure $1, \mathrm{C}_{42} \mathrm{H}_{72} \mathrm{O}_{14}$, molecular weight $=801.01$, purity by high-performance liquid chromatography (HPLC) $\geq 98 \%$ ) was purchased from Solarbio (China), and z-YVAD-FMK $\left(\mathrm{C}_{31} \mathrm{H}_{39} \mathrm{FN}_{4} \mathrm{O}_{9}, \mathrm{MW}\right.$ : 630.66, purity: $\geq 98 \%$ ) from ApexBio Technology LLC (USA). Streptozotocin (STZ), 4-methyl-N1-(3-phenylpropyl)-1,2-benzenediamine (JSH-23) and L-leucine (LEU, $\mathrm{C}_{6} \mathrm{H}_{13} \mathrm{NO}_{2}, \mathrm{MW}: 131.17$, purity: $\geq 98 \%$ ) were purchased from Sigma-Aldrich (St. Louis, MO, USA). Rapamycin was bought from Selleck Chemicals. Sodium palmitate was purchase from Mecen Unicreate (Beijing, China).

2.2. Cell Culture. The conditionally immortalized mouse podocyte cell line BNCC337685 was obtained from BeNa Culture Collection (Beijing, China). The cells were cultured in low glucose DMEM (Genview, Florida, USA) supplemented with $10 \%$ fetal bovine serum (FBS; Gibco, Carlsbad, CA, USA) and recombinant IFN- $\gamma$ (PeproTech, London, $\mathrm{UK}$ ) at $33^{\circ} \mathrm{C}$ for proliferation, and without IFN- $\gamma$ at $37^{\circ} \mathrm{C}$ for more than 7 days for inducing differentiation. The cells were harvested at $\sim 80 \%$ confluency and maintained in serum-free conditions for the different assays.

2.3. Proliferation Assay. The podocytes were seeded in 96well plates in serum-free DMEM at the density of $8 \times 10^{4}$ per well and cultured for $24 \mathrm{~h}$. Hyperlipidemia was induced with $100 \mu \mathrm{M}$ palmitate (PA) in the absence or presence of 0,10 , $25,50,75$, or $100 \mu \mathrm{M}$ Rg1 for $48 \mathrm{~h}$ [28]. The suitably treated cells were then incubated for an additional $2 \mathrm{~h}$ with cellcounting kit-8 (CCK-8, KeyGEN BioTECH, Nanjing, China) solution $(10 \mu \mathrm{L} /$ well $)$, and the optical density of each well was measured at $450 \mathrm{~nm}$. A decrease in cell viability to $30-40 \%$ was indicative of podocyte injury $[29,30]$.

2.4. Small Interfering RNA (siRNA) Transfection. The NLRP3-specific (si-NLRP3) and scrambled siRNAs (si-con) were designed and synthesized by RiboBio Co. Ltd. The

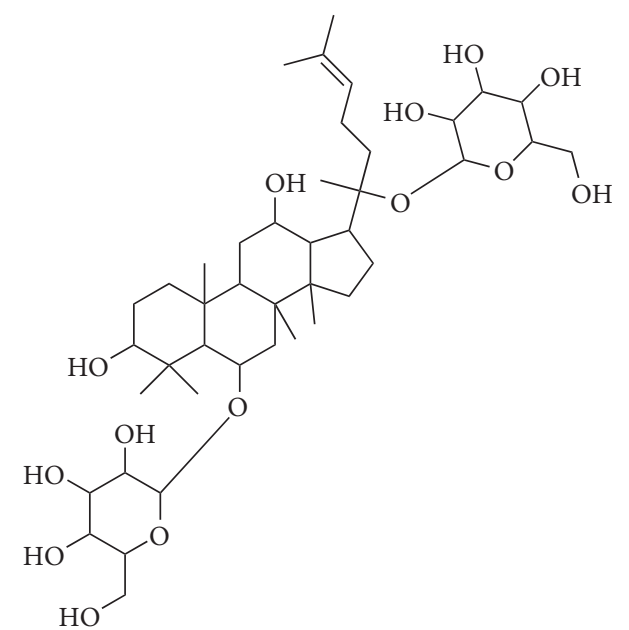

Figure 1: Chemical structure of Ginsenoside Rg1.

podocytes were transfected according to the manufacturer's protocol and analyzed for NLRP3 levels later. The NLRP3targeting siRNA sequence was GTACTTAAATCGTGAAACA.

2.5. Establishment of DN Model. SPF-grade male SpragueDawley rats (aged 8 weeks, weighing 180-200 g) were purchased from the Beijing Vital River Laboratory Animal Technology Co. Ltd. Diabetes was induced by intraperitoneally injecting the animals once with $50 \mathrm{mg} / \mathrm{kg} \mathrm{STZ} \mathrm{(Sigma-}$ Aldrich, St. Louis, MO, USA) in sodium citrate buffer. The placebo/normal controls (NC, $n=8$ ) were injected with an equal volume of $0.1 \mathrm{M}$ citrate buffer, $\mathrm{pH}$ 4.5. Serum glucose levels were measured 3 days after injection after drawing blood from the tail veins. Fasting glucose levels $\geq 16.7 \mathrm{mM}$ for 3 consecutive days were indicative of diabetes [31, 32]. The diabetic rats were fed the high fat diet (HFD; $10 \%$ lard, $20 \%$ sucrose, $2.5 \%$ cholesterol, $0.5 \%$ sodium cholate, and $67 \%$ basic feed) for 4 weeks to induce DN, while the control rats were given normal food. Four weeks after STZ injection, the successfully modelled $\mathrm{DN}$ rats were randomly divided into the (untreated) DN and ginsenoside Rg1-treated groups ( $n=8$ in each group) and administered with vehicle (aqua distillate) or $50 \mathrm{mg} / \mathrm{kg}$ ginsenoside $\mathrm{Rg} 1$ once daily by oral gavage for 8 weeks. This dose of ginsenoside Rg1 has been proved to be effective and safe [33]. At the end of the regimen, the rats were anaesthetized adequately; then, the urine creatinine (UCr), urinary microalbumin (Malb), blood urea nitrogen (BUN), and serum creatinine (Scr) levels were measured. The rats were then euthanized, and their renal cortices were harvested for further analyses. All animal experiments were approved by the Institutional Animal Care and Use Committee at Capital Medical University and conformed to the Guidelines for the Care and Use of Laboratory Animals by the National Institute of Health.

2.6. Histological Analysis. Freshly extracted renal tissues were fixed in $4 \%$ paraformaldehyde (KeyGEN BioTECH, Nanjing, China), embedded in paraffin, and cut into $4 \mu \mathrm{m}$ thick sections. Hematoxylin-eosin (HE), periodic acid-Schiff 
(PAS), and Masson's staining were performed as per standard protocols, and the tissue sections were observed under a light microscope (Leica DM60008, Kyoto, Japan). For transmission electron microscopy (TEM), the renal cortices were fixed in $2 \%$ glutaraldehyde (Servicebio, Wuhan, China), cut into ultrathin sections, and stained accordingly [34]. The samples were observed under a TEM (JEM-1400 plus, JEOL, Tokyo, Japan).

2.7. Biochemical Assays. The levels of serum creatinine, urine creatinine, and urine albumin were measured using specific kits from Leadman Biochemistry (Beijing, China) in accordance with the manufacturer's instructions. The biochemical parameters were detected with an automatic chemistry analyzer (7600, HITACHI, Tokyo, Japan). The urinary protein content was calculated relative to the creatinine levels.

2.8. Immunofluorescence (IF) and Phalloidin Staining. The paraffin sections were deparaffinized, dehydrated, and heated in citrate buffer for antigen retrieval. After blocking with $10 \%$ goat serum (ZSGB-BIO, Beijing, China) for $1 \mathrm{~h}$, the sections were incubated overnight with rabbit antinephrin (1:50, sc-377246, Santa Cruz Biotech, CA, USA) antibody and rabbit anti-caspase-1 antibody $\left(1: 200\right.$, ab1872) at $4^{\circ} \mathrm{C}$, followed by FITCconjugated goat anti-rabbit secondary antibody $(1: 100$; clone ZF-0311, ZSGB-BIO, Beijing) for $1 \mathrm{~h}$ at $37^{\circ} \mathrm{C}$. The sections were counterstained with $4^{\prime}, 6^{\prime}$-diamidino-2-phenylindole (DAPI) (Genview, Florida, USA) and imaged using a laser confocal microscope (TCS SP8 STED, Leica, Wetzlar, Germany). Alternatively, the podocytes were permeabilized with Triton $\mathrm{X}-100$, washed thrice with PBS, and stained with $100 \mu \mathrm{g} / \mathrm{mL}$ phalloidin-conjugate working solution (AAT Bioquest, Sunnyvale, CA, USA) for $1 \mathrm{~h}$ at room temperature. The stained cells were washed thrice with PBS and observed under a laser scanning confocal microscope.

2.9. Immunohistochemical Staining. The paraffin-embedded sections were processed via routine dewaxing and hydration. In brief, sections were heated in a microwave for antigen retrieval and blocked with $3 \% \mathrm{H}_{2} \mathrm{O}_{2}$ (ZSGB-BIO, Beijing, China) for $10 \mathrm{~min}$ to avoid nonspecific binding. The sections were subsequently incubated with anti-ASC antibody (1: $100, a b 47092$, abcam, UK) at $4^{\circ} \mathrm{C}$ overnight and reacted with poly peroxidase-anti-mouse IgG (ZSGB-BIO, Beijng, China). Afterwards, 3,3-diaminobenzidine (DAB, ZSGBBIO) was used for visualization of immunoreactive signals. Finally, the sections were observed under a microscope (Leica DM60008) at 400x magnifications.

2.10. TUNEL Assay. Tissue sections and podocytes cultured on coverslips were treated with proteinase $\mathrm{K}(20 \mathrm{~g} / \mathrm{mL})$ and stained using the In-Situ Cell Death Detection kit (Nanjing KeyGen Biotech Co. Ltd., China) according to the manufacturer's instructions. The number of TUNEL-positive cells was counted in 10 randomly selected, nonoverlapping fields at 200x magnification. Apoptotic index was calculated as the number of TUNEL-positive cells per $10^{3}$ cells and \%TUNEL-positive podocytes.

2.11. Western Blotting. The total protein of cultured podocytes and kidney tissues were extracted, separated by SDS-PAGE, and then transferred to PVDF membranes. After blocking with 5\% skim milk in PBS $+0.05 \%$ tween 20 , the blots were incubated overnight with rabbit polyclonal antibodies against procaspase1 (1:1000, ab179515), IL-1 $\beta(1: 500$, ab9722), mTOR $(1: 1000$, ab2732), NLRP3 (1:500, ab214185), ASC (1:500, ab47092), caspase-1 p20 (Casp1 p20, 1:500, ab1872) (all from Abcam, Cambridge, UK) and p-mTOR $(1: 500,5536)$, rabbit monoclonal antibodies targeting NF- $\kappa$ B p65 (p-65, $1: 1000,8242)$ and p-NF- $\kappa$ B p65 (p-p65, 1:500, 3033) (all from Cell Signaling Technology, Inc., Danvers, MA, USA), and mouse monoclonal antinephrin $(1: 500, s c-377246)$ and anti-GAPDH $(1: 2000$, 60004-1-lg; Proteintech Group, Rosemont, USA) antibodies at $4^{\circ} \mathrm{C}$. The blots were then incubated with HRP goat anti-rabbit IgG (RS0002) or HRP goat anti-mouse IgG (RS0001) secondary antibodies (both diluted 1:5000, ImmunoWay Biotechnology, TX, USA) as appropriate. The positive bands were developed using a chemiluminescent reagent (Thermo Scientific, USA).

\subsection{Reverse Transcription Quantitative Polymerase Chain} Reaction (RT-qPCR). Total RNA was isolated from the cultured podocytes and renal cortices using TRIzol Reagent according to the manufacturer's instructions and reversetranscribed using the PrimeScript ${ }^{\mathrm{TM}}$ RT kit. The qRT-PCR analysis was performed using the $\mathrm{SYBR}^{\circledR}$ Premix $\mathrm{Ex} \mathrm{Taq}^{\mathrm{TM}} \mathrm{II}$ (Takara) in the ABI PRISM 7500 FAST Real-TIME PCR System (ABI, Vernon, CA, USA). Relative gene expression levels were calculated by the $2 \Delta \mathrm{CT}$ method. The sequence of mTOR primers was as follows: forward: $5^{\prime}$-AGTGAAGCCGAGAGCAATGAGA-3' and reverse: $5^{\prime}$ GACAAGGAGATAGAACGGAAGAAGC-3' .

\subsection{Propidium Iodide (PI)/Hoechst 33342 Double Staining.} The suitably treated podocytes were harvested and stained with Hoechst 33342 at $37^{\circ} \mathrm{C}$ for 10 minutes. The cells were washed with PBS 3 times, and then the $1 \times$ Buffer and the PI were added to stain the podocytes in the dark for 10 minutes. The cells were observed under an inverted fluorescence microscope (Carl Zeiss, Oberkochen, Germany). Whether the PI could penetrate the pyroptosis cell membrane and enter into the nucleus was determined by the appearance of red fluorescence, indicating the presence of dead cells as a result of pyroptosis. The pyroptosis rate was calculated as the number of dead cells/the total number of cells $\times 100 \%$. The experiment was conducted in triplicate.

2.14. Statistical Analysis. The data were presented as mean\pm standard deviation (SD). Statistical analysis was performed using the SPSS 19.0 software (IBM Corporation, Armonk, NY, USA). Student's $t$-test was used to compare the difference between two groups, while multiple groups were compared by one-way analysis of variance (ANOVA). A $P$ value $<0.05$ was considered statistically significant. 

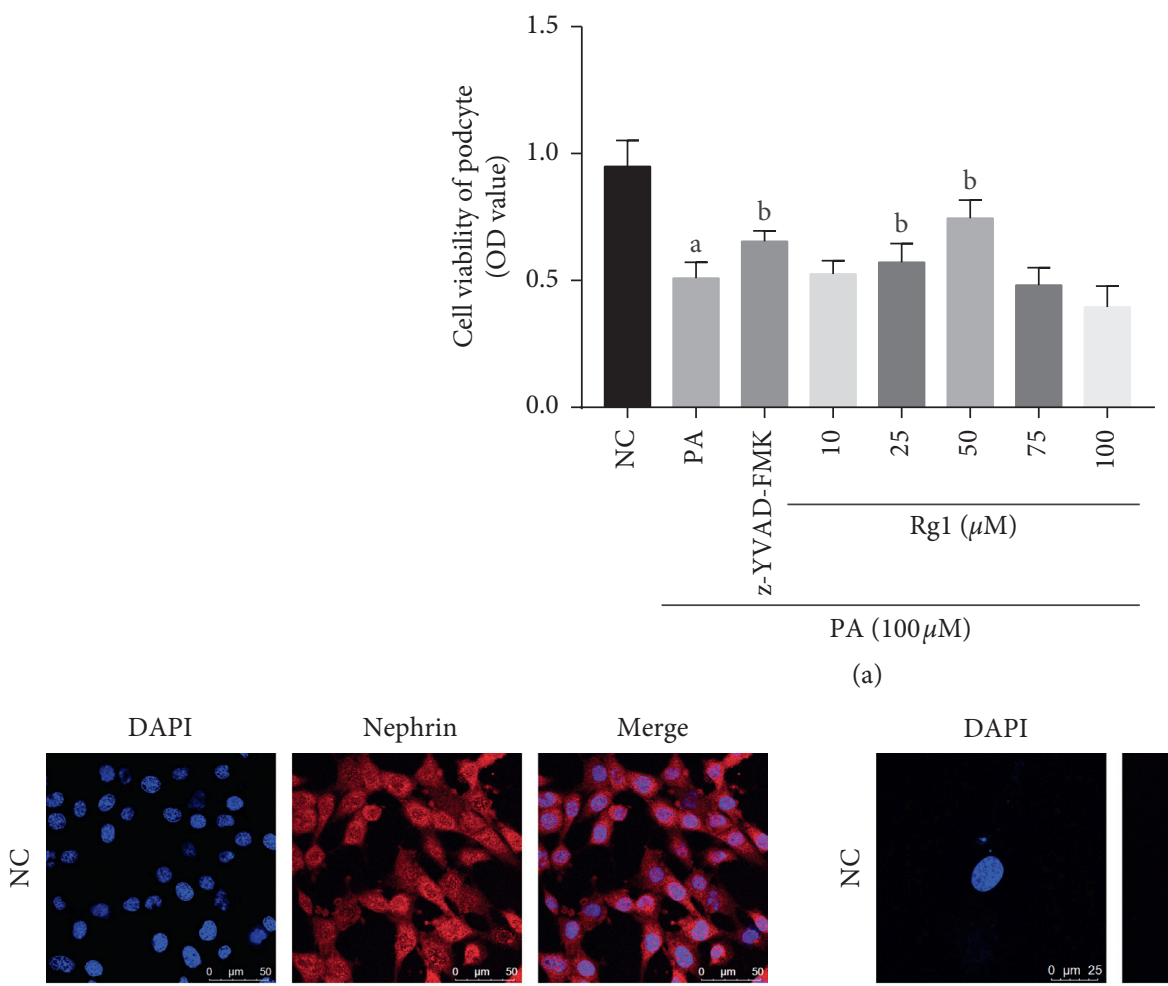

(a)
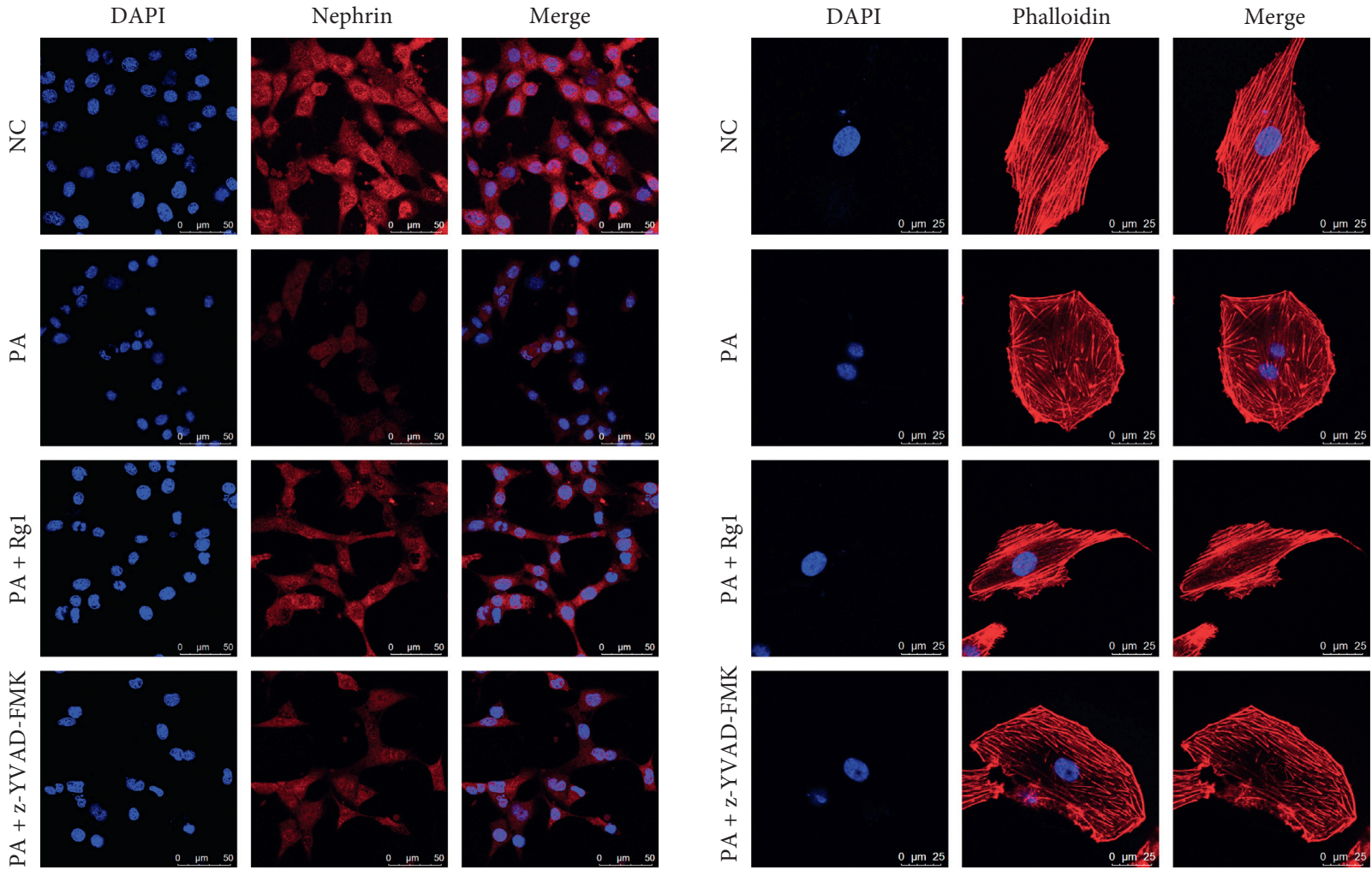

(b)
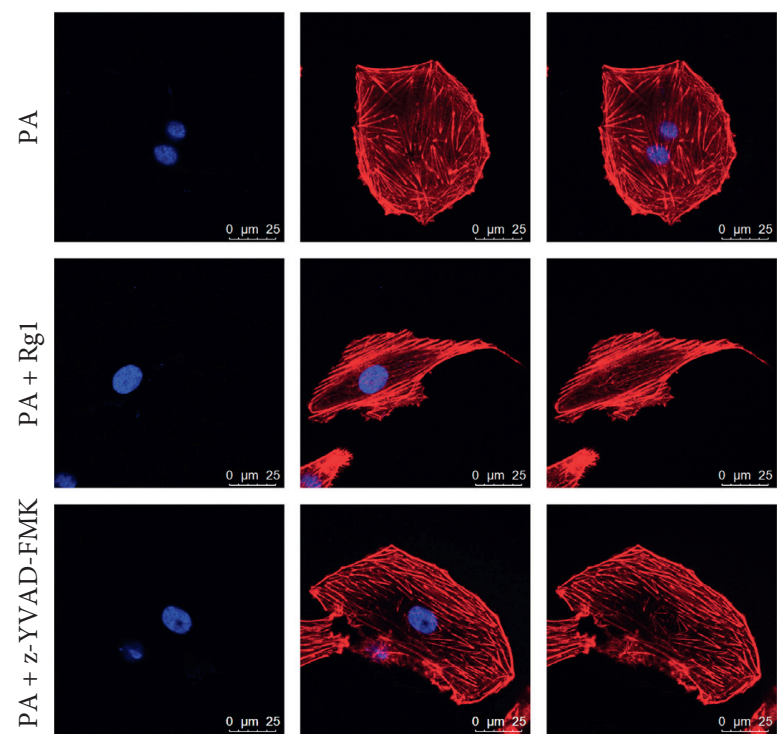

(c)

Figure 2: Protective effect of $\operatorname{Rg} 1$ on hyperlipidemia-treated podocytes. (a) The proliferation rate of podocytes exposed to hyperlipidemic conditions with/without Rg1 or z-YVAD-FMK for $48 \mathrm{~h}$. (b) Representative images of immunofluorescent staining showing nephrin expression in cultured podocytes (original magnification 630x). (c) Representative images of podocytes with phalloidin-stained cytoskeleton (original magnification 630x). Values are the mean $\pm \mathrm{SD} ; n=3$. ${ }^{\mathrm{a}} \mathrm{P}<0.05$ compared to the NC group, ${ }^{\mathrm{b}} P<0.05$ compared to the $\mathrm{PA}$ group. Abbreviations: PA, palmitate group; Rg1, ginsenoside Rg1 group; NC, normal control; DN, diabetic nephropathy group; DAPI, 4',6diamidino-2-phenylindole.

\section{Results and Discussion}

3.1. Ginsenoside Rg1 Protects Podocytes against Hyperlipidemia-Induced Injury. The optimal therapeutic dose of Rg1 was determined in an in vitro model of podocyte injury. As shown in Figure 2(a), Rg1 increased the viability of podocytes in a dose-dependent manner at lower concentrations for $48 \mathrm{~h}$, while doses above $50 \mu \mathrm{M}$ were cytotoxic. Therefore, $50 \mu \mathrm{M} \operatorname{Rg} 1$ for $48 \mathrm{~h}$ was used for subsequent experiments. Podocytes exposed to hyperlipidemic conditions showed reduced activity and low expression level of nephrin, while these effects were restored by Rg1 treatment (Figures 2(a) and 2(b)). Consistent with the above observation in vitro, in normal group rats, nephrin staining presented a green 

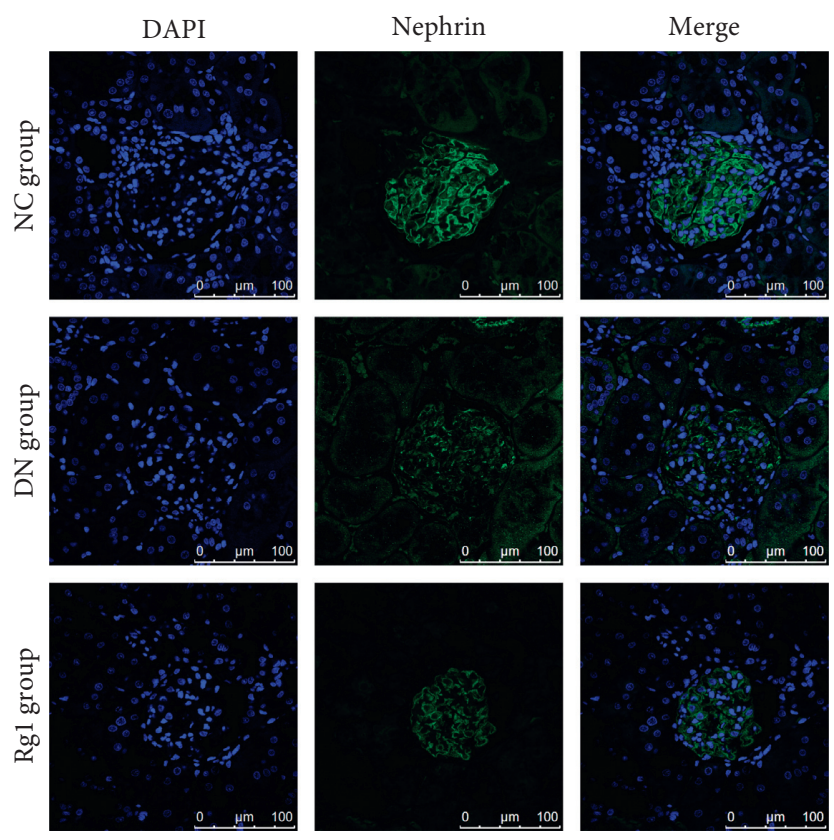

FIGURE 3: Effect of Rg1 on nephrin expression of DN rats: representative images of immunofluorescent staining showing in situ nephrin expression in renal tissues. Blue, nuclear staining (DAPI); green, target protein staining (original magnification $630 \mathrm{x}, n=3$ ).

linear-like pattern along the capillary loops of glomeruli. Instead, a light green, discontinuous, short linear-like or punctiform staining pattern of nephrin was observed in DN group, and Rg1 also upregulated the in situ expression of nephrin in the glomeruli of DN rats (Figure 3).

In vitro, actin stress fibers were arranged regularly or parallel to each other in podocytes of normal group. Phalloidin staining further revealed the collapsed or totally absent intracellular actin stress fibers in the hyperlipidemiatreated podocytes, resulting in the polygonal cells. Ginsenoside $\mathrm{Rg} 1$ treatment remodeled the podocyte skeleton and restored their normal shape (Figure 2(c)).

Taken together, Rg1 protects podocytes against hyperlipidemia-induced injury by restoring nephrin levels and the cytoskeleton.

\subsection{Ginsenoside Rg1 Alleviated Hyperlipidemia-Induced} Pyroptosis in Podocytes. The positive rate of PI staining revealed that, compared with the NC group, there was significant increased pyroptosis rate in PA-induced podocytes (Figures 4(a) and 4(b)). Pyroptosis is also characterized by caspase-1 activation and IL-1 $\beta$ secretion [35]. As shown in Figures $4(\mathrm{c})$ and $4(\mathrm{~d})$, the expression of Casp $1 \mathrm{p} 20$ and IL-1 $\beta$ was markedly increased in the hyperlipidemia-stressed podocytes. Consistent with this, pretreatment of the hyperlipidemiastressed podocytes with $20 \mu \mathrm{M}$ caspase-1 inhibitor z-YVADFMK significantly increased cell viability and nephrin levels, decreased the rate of PI-positive podocytes, and restored the cytoskeletal structure (Figures 2(a)-2(c), 4(a) and 4(b)), thus further confirming that hyperlipidemia-induced podocyte injury in vitro was partly mediated via pyroptosis induction. Furthermore, Rg1 significantly decreased pyroptosis rates in the suitably treated podocytes, which was also correlated to markedly lower levels of Casp1 p20 and IL-1 $\beta$ compared to the
PA group (Figures 4(a)-4(d)). However, the pyroptosis rate and the level of Casp1 p20 of Rg1 group were higher than those in the $\mathrm{PA}+\mathrm{z}-\mathrm{YVAD}-\mathrm{FMK}$ groups; these results also strongly suggested that hyperlipidemia induced pyroptosis of podocytes, which was alleviated by Rg1.

Moreover, increased TUNEL-positive cells were observed in the glomeruli and renal tubules of the DN rats, which was significantly alleviated by Rg1 treatment (Figures 5(a) and 5(b)). Immunofluorescence images and immunoblot showed that the glomeruli of the untreated $\mathrm{DN}$ rats also expressed higher levels of activated caspase-1 (Casp1 p20) and IL-1 $\beta$, which was decreased by $\operatorname{Rg} 1$ (Figures 5(c) and 5(d)).

\subsection{Ginsenoside Rg1 Targets the mTOR/NF- $\kappa B$ Pathway in} Podocytes under Hyperlipidemic Conditions. Studies have implicated mTOR in podocytes injury and DN $[36,37]$. Consistent with this, hyperlipidemia treatment significantly increased total and phosphorylated mTOR levels in the cultured podocytes (Figures 6(a) and 6(b)), as well as in renal tissues of diabetic rats (Figure 7). Furthermore, pretreatment with the mTOR inhibitor rapamycin $(10 \mathrm{ng} / \mathrm{mL})$ decreased total and phosphorylated mTOR levels (Figures 6(a) and $6(\mathrm{~b})$ ), inhibited the expression of Casp1 p20 and IL-1 $\beta$, and also abrogated hyperlipidemia-induced pyroptosis (Figure 4). Rg1 significantly downregulated $\mathrm{mTOR}$ and p-mTOR (Figure 6(a)), as well as p-p65 (Figure 6(c)) in the hyperlipidemic podocytes. However, pretreatment of these podocytes with the mTOR activator LEU $(5 \mathrm{mM})$ abrogated the suppressive effects of Rg1 on p-p65 (Figure 6(c)). Therefore, the mTOR/NF- $\kappa$ B pathway may be one of the effect targets of $\mathrm{Rg} 1$ on the PA-induced podocytes.

Similarly, mTOR and p-p65 were significantly upregulated in the renal tissues of untreated DN rats compared to the $\mathrm{NC}$ group and decreased following Rg1 treatment (Figure 7). 


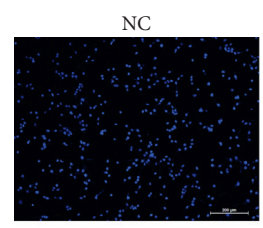

$\mathrm{PA}+\mathrm{Rapa}$
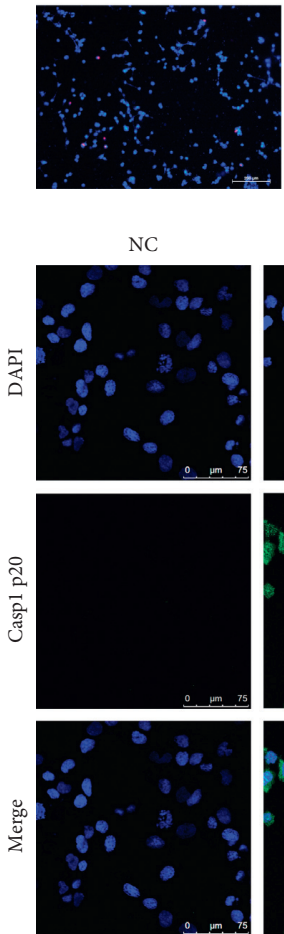

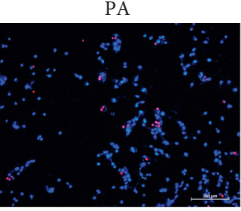

PA + z-YVAK-FMK

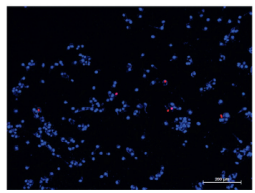

(a)
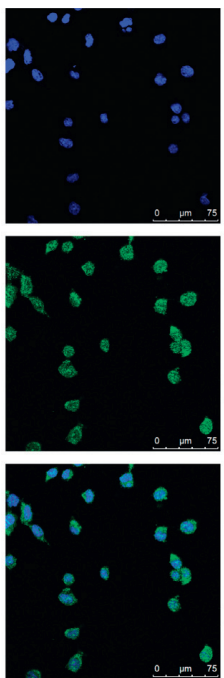
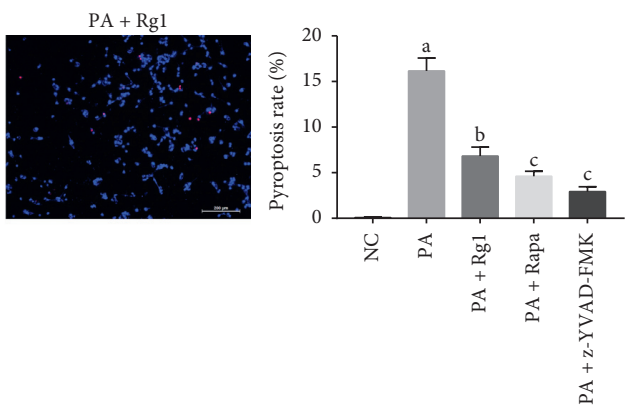

$\mathrm{PA}+\operatorname{Rg} 1$
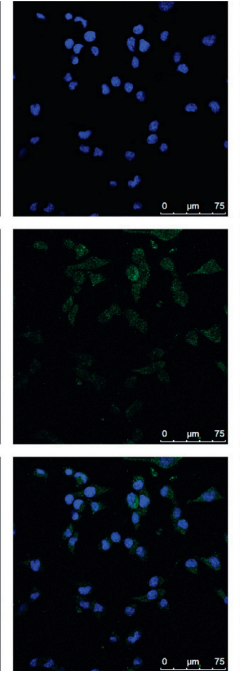

PA + Rapa
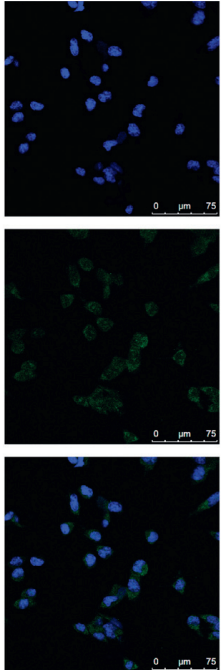

$\mathrm{PA}+\mathrm{z}$-YVAD-FMK
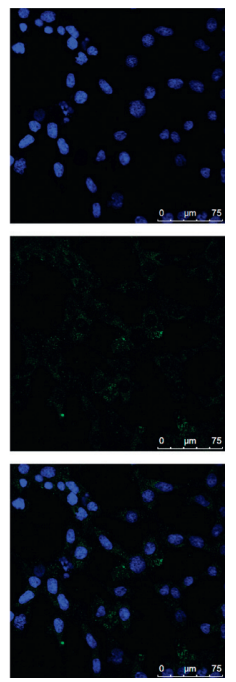

(c)
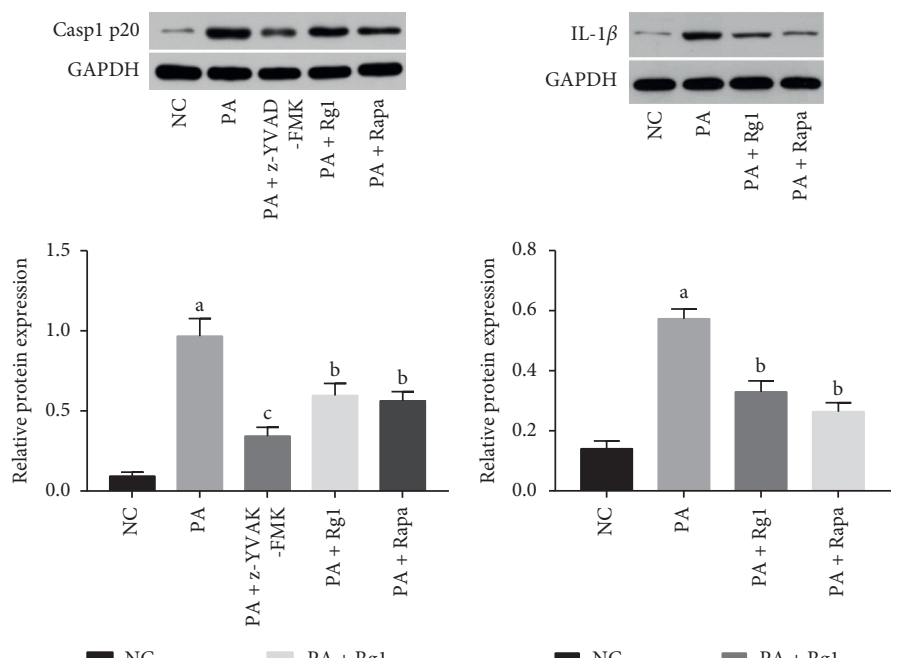

$$
\begin{array}{ll}
\text { NC } & \text { PA + Rgl } \\
\text { PA } & \text { PA+ Rapa } \\
\text { PA + z-YVAK } & \\
\text {-FMK } &
\end{array}
$$

$$
\begin{array}{ll}
\mathrm{NC} & \mathrm{PA}+\mathrm{Rg} 1 \\
\mathrm{PA} & \mathrm{PA}+\mathrm{Rapa}
\end{array}
$$

Figure 4: Effect of Rg1 on hyperlipidemia-induced pyroptosis in podocytes. (a)-(b) Representative PI/Hoechst 33342-stained images showing pyroptotic podocytes. Red fluorescence was regarded as PI-positive podocytes, indicating the presence of dead cells as a result of pyroptosis (original magnification 200x). (c) Representative immunofluorescent images showing in situ Casp1 p20 expression in the suitably treated podocytes (original magnification 630x). (d) Immunoblot showing total Casp1 p20 and IL-1 $\beta$ levels in the suitably treated podocytes. Values are the mean $\pm \mathrm{SD} ; n=3$. ${ }^{\mathrm{a}} P<0.05$ compared to the NC group, ${ }^{\mathrm{b}} P<0.05$ compared to the PA group, ${ }^{\mathrm{c}} P<0.05$ compared to the PA + Rg1 group. Abbreviations: Rapa, rapamycin. 


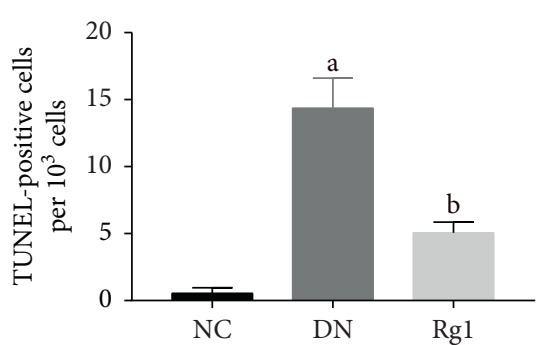

(a)

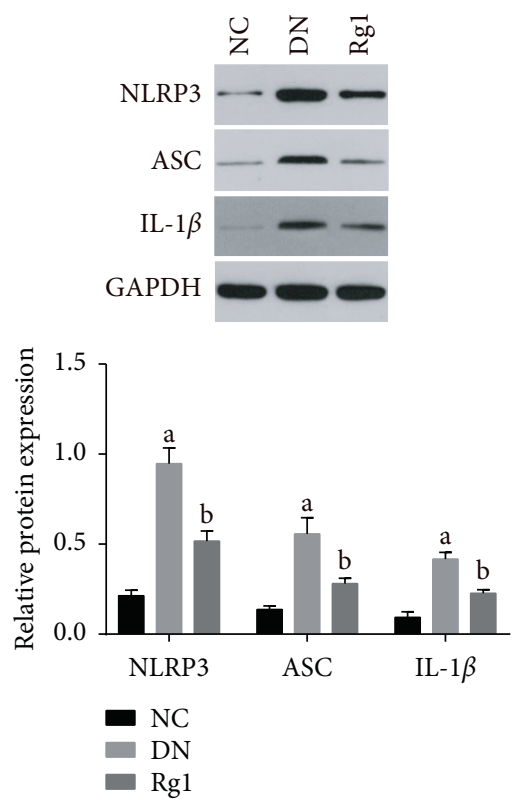

(c)

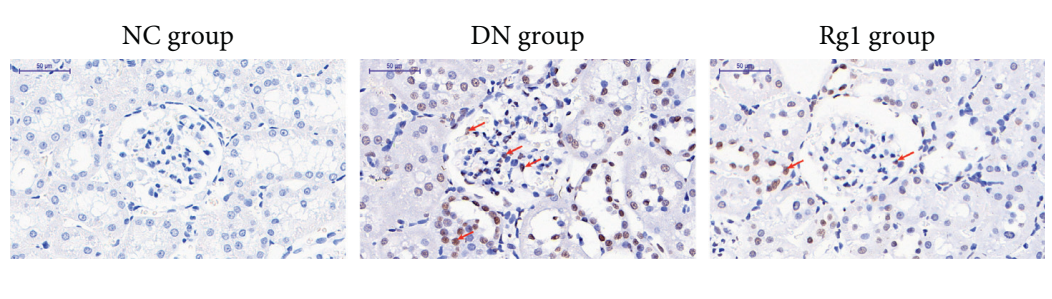

(b)
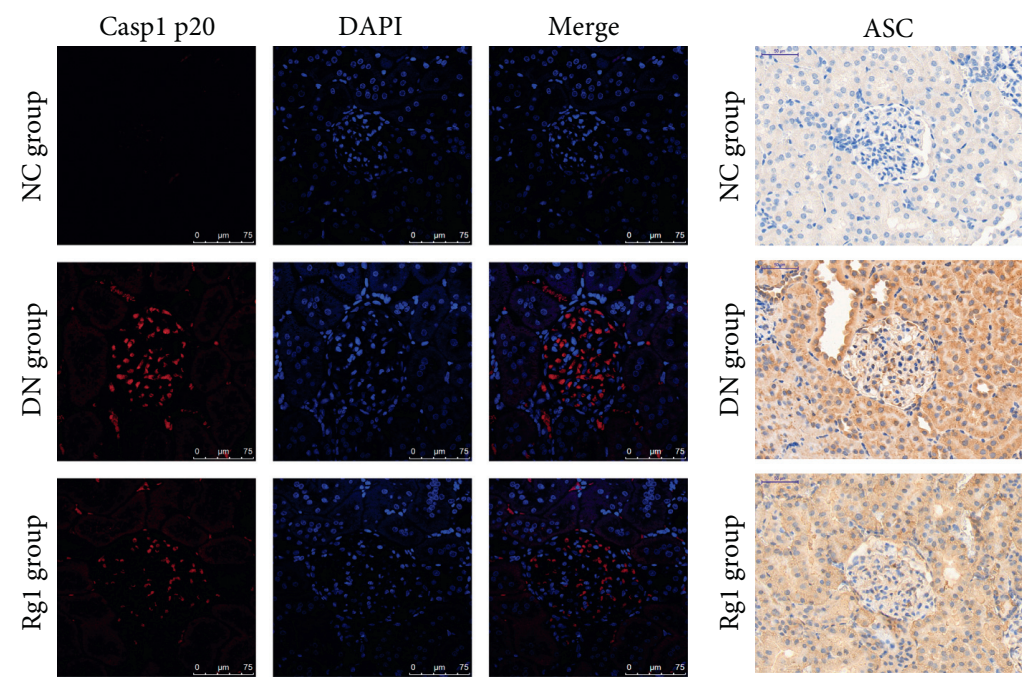

(d) (e)

FIGURE 5: Rg1 inhibited NLRP3 and alleviated cell death in diabetic renal tissues. (a)-(b) Representative TUNEL images showing apoptotic cells in the renal tissue (original magnification 400x); red arrows point to the TUNEL-positive cells. (c) Immunoblot showing NLRP3, ASC, and IL-1 $\beta$ levels in the renal tissues of the different groups. (d) Representative immunofluorescent images showing in situ Casp1 p20 expression in renal tissues. Blue, nuclear staining (DAPI); red, target protein staining (original magnification 630x). (e) Representative immunohistochemical staining showing in situ ASC expression in renal tissues. Brownish or yellowish deposits were regarded as positive immunostaining (original magnification $400 \mathrm{x}$ ). Values are the mean $\pm \mathrm{SD} ; n=3 .{ }^{\mathrm{a}} \mathrm{P}<0.05$ compared with the NC group, ${ }^{\mathrm{b}} \mathrm{P}<0.05$ compared with the PA group. Abbreviations: NLRP3, nucleotide-binding oligomerization domain-like receptor with a pyrin domain (NLRP) 3; ASC, apoptosis-associated speck-like protein.

3.4. Rg1 Inhibited Hyperlipidemia-Induced NLRP3 in Podocytes by Suppressing the $m T O R / N F-\kappa B$ Pathway. Since several inflammasomes can trigger pyroptosis, we silenced NLRP3 in the podocytes in order to determine whether NLRP3 inflammasome is essential for hyperlipidemia-induced pyroptosis in podocytes. As shown in Figure 8, NLRP3 knockdown (Figure 8(a)) significantly reduced the pyroptosis rate (Figure 8(c)) and the expression of Casp1 p20 and IL-1 $\beta$ in the podocytes exposed to hyperlipidemic conditions (Figure $8(b)$ ). This suggests that podocyte pyroptosis induced by hyperlipidemia was mainly regulated by NLRP3 inflammasome.

Previous studies have shown that NLRP3 is activated by mTOR/NF- $\kappa \mathrm{B}[13,38]$. Consistent with this, the NF- $\kappa \mathrm{B}$ inhibitor JSH-23 $(20 \mu \mathrm{M})$ and rapamycin significantly decreased the expression of ASC and Casp1 p20 in the hyperlipidemic podocytes (Figure 9), which were significantly elevated by LEU.
Interestingly, JSH-23 neutralized the activation effects of LEU on NLRP3 under hyperlipidemic conditions (Figure 9). Furthermore, $\operatorname{Rg} 1$ markedly inhibited the expression and activation of NLRP3 under hyperlipidemic conditions; however, the inhibitory effect of Rg1 on NLRP3 was attenuated by LEU (Figure 9). NLRP3, ASC, and Casp1 p20 levels were also upregulated in the glomeruli of DN rats and deceased upon $\mathrm{Rg} 1$ treatment (Figures 5(c)-5(e)). These findings indicated that hyperlipidemia-induced NLRP3 activation was dependent on the mTOR/NF- $\kappa$ B pathway, and Rg1 targeted the mTOR/NF$\kappa \mathrm{B} / \mathrm{NLRP} 3$ axis to alleviate pyroptosis in the podocytes.

3.5. Effect of Ginsenoside Rg1 on Renal Function and Morphology in Diabetic Rats. The therapeutic effects of ginsenoside Rg1 on renal function and morphology were measured in terms of standard biochemical and histopathological indices. Rg1 

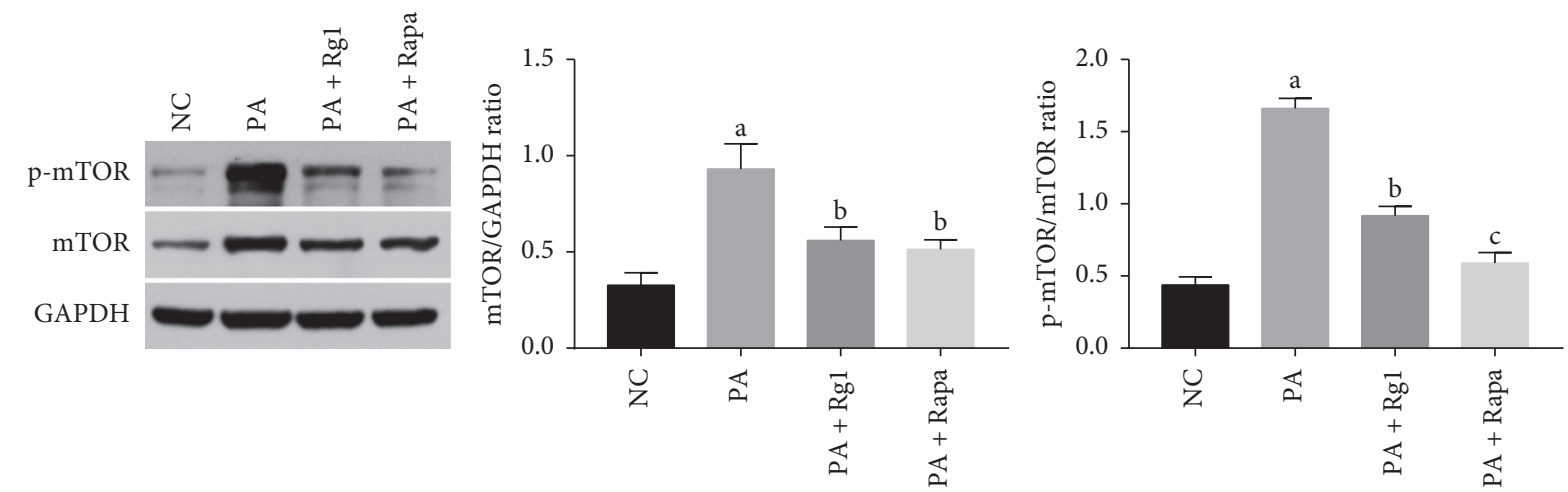

(a)

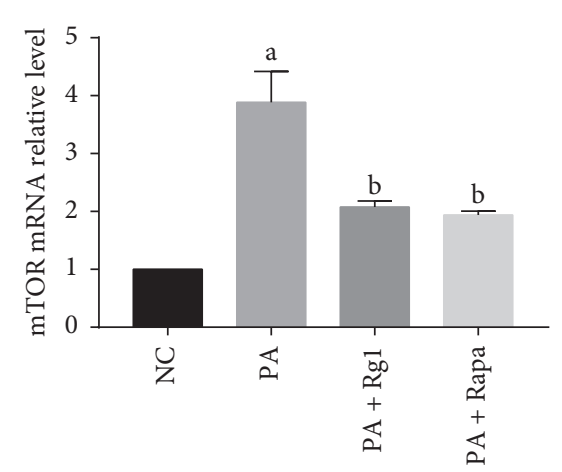

(b)

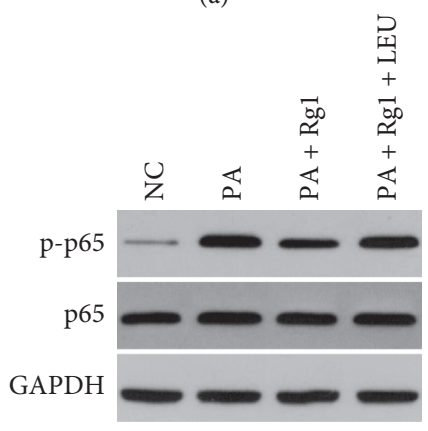

(c)

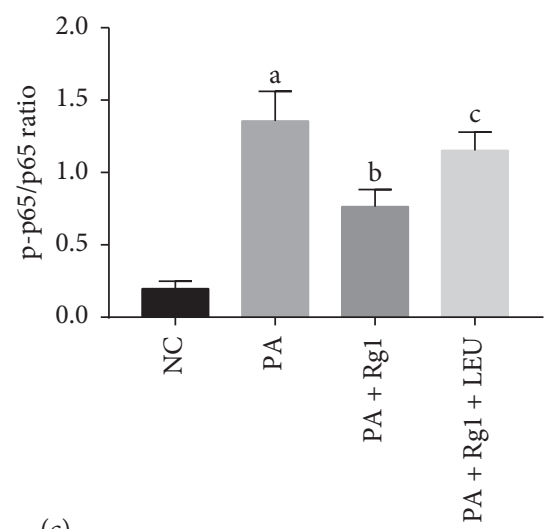

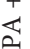

FIgURE 6: Rg1 inhibits mTOR and NF- $\kappa$ B p65 expression in the podocytes in vitro. (a) Immunoblot showing mTOR and p-mTOR levels in the suitably treated podocytes. (b) The mTOR mRNA levels in the podocytes treated as indicated. (c) Immunoblot showing p-p65 and p65 levels in the suitably treated podocytes. Values are the mean $\pm \mathrm{SD} ; n=3 .{ }^{\mathrm{a}} P<0.05$ compared with the NC group, ${ }^{\mathrm{b}} P<0.05$ compared with the PA group, ${ }^{\mathrm{c}} \mathrm{P}<0.05$ compared with the $\mathrm{PA}+\mathrm{Rg} 1$ group.
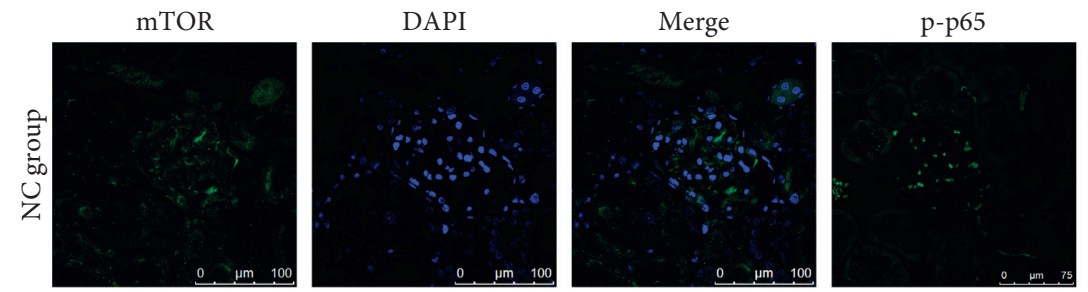

DAPI
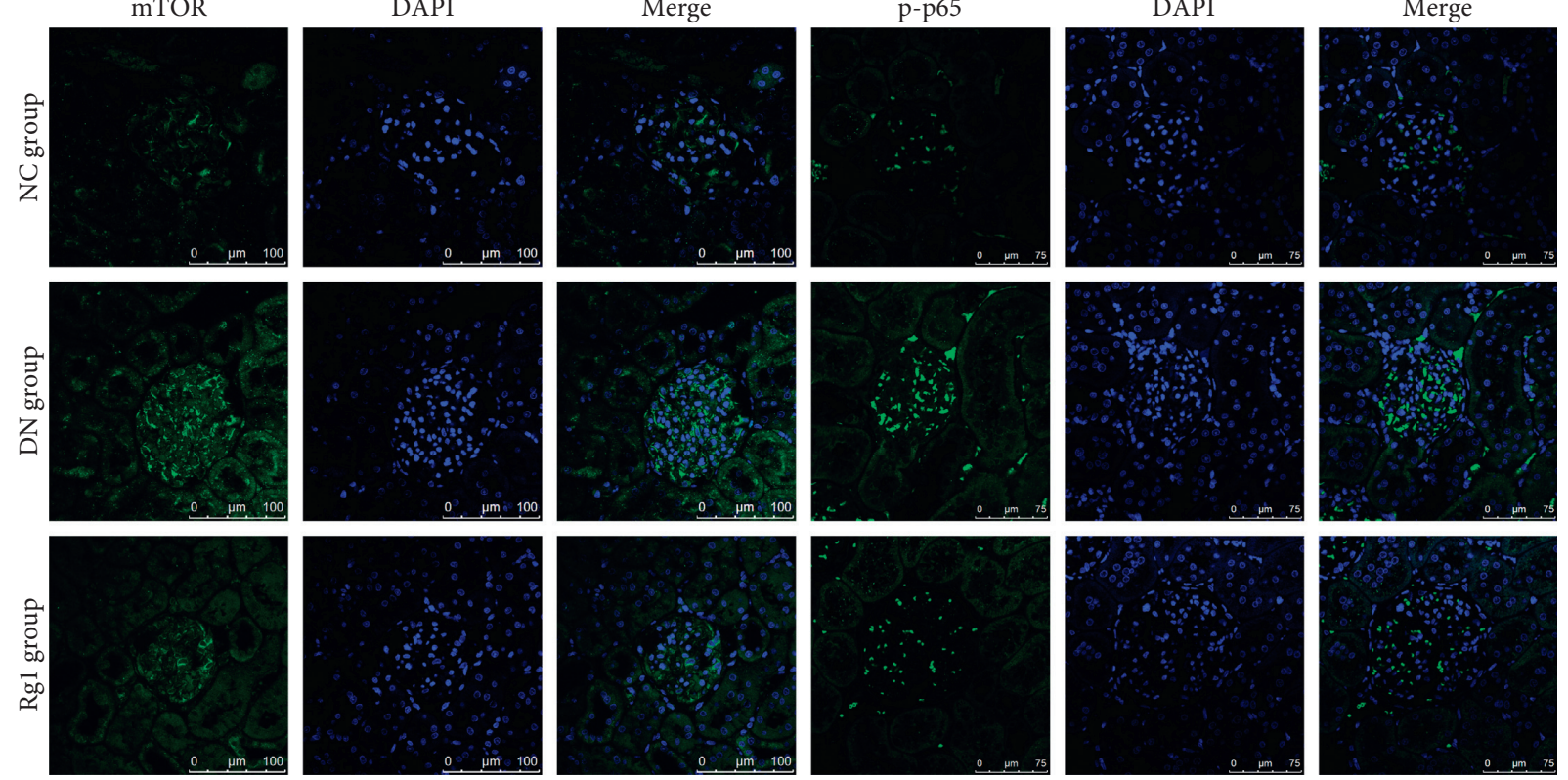

FIgURE 7: Rg1 inhibits mTOR and NF- $\kappa$ B p65 expression in diabetic renal tissues. Representative immunofluorescence images showing in situ mTOR and p-p65 expression in the renal tissues. Blue, nuclear staining (DAPI); green, target protein staining (original magnification $630 \mathrm{x}, n=3)$. 

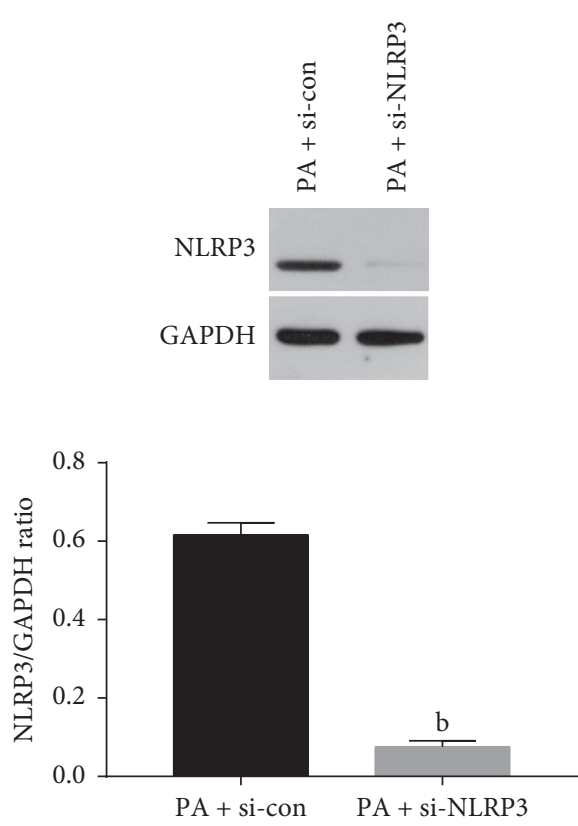

(a)
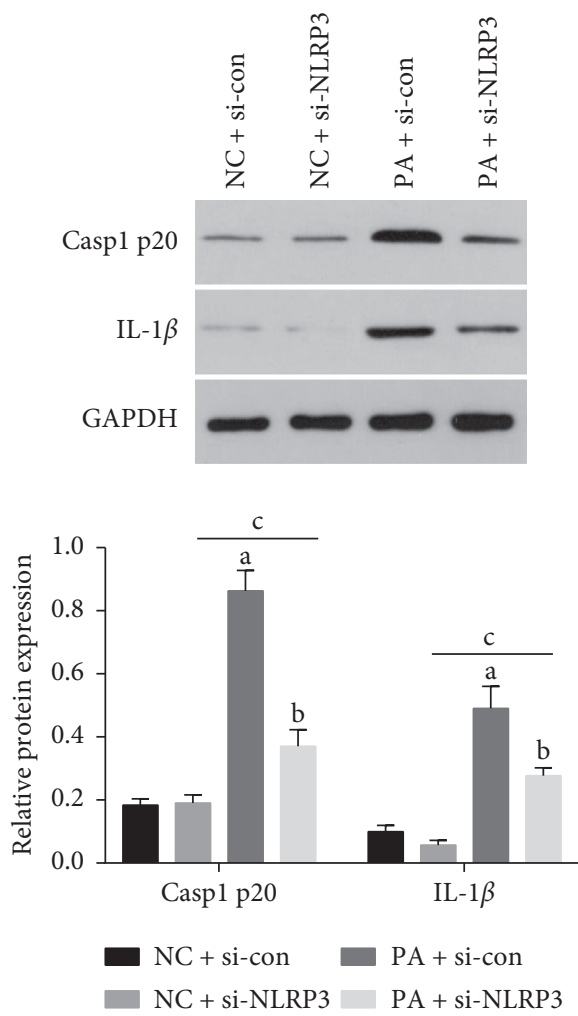

(b)
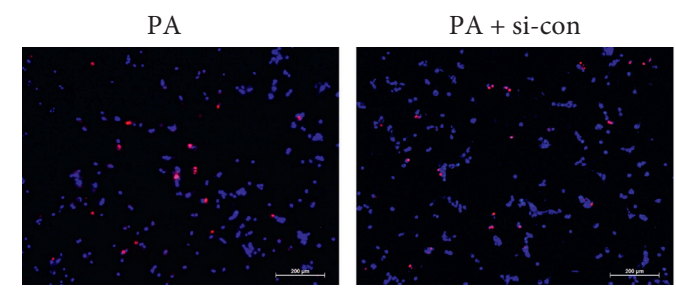

(c)
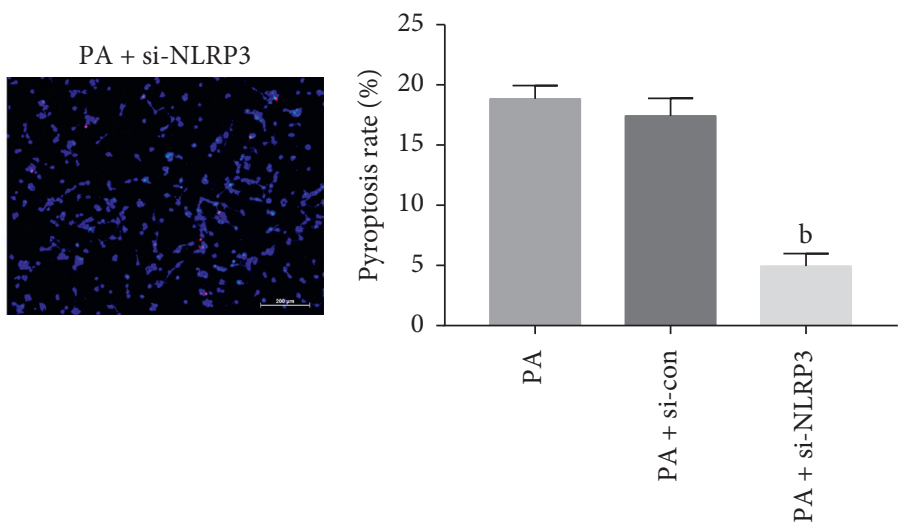

(d)

FIGURE 8: Downregulation of NLRP3 inhibited pyroptosis in podocytes under hyperlipidemic conditions. The podocytes were treated with si-con or si-NLRP3 in the presence (or absence) of PA for $48 \mathrm{~h}$. (a) NLRP3 and (b) Casp1 p20 and IL-1 $\beta$ levels in the suitably treated cells. (c)-(d) Representative PI/Hoechst 33342-stained images showing pyroptotic podocytes (original magnification 200x). Values are the mean $\pm \mathrm{SD} ; n=3$. ${ }^{\mathrm{a}} \mathrm{P}<0.05$ compared with the $\mathrm{NC}+$ si-con group,${ }^{\mathrm{b}} \mathrm{P}<0.05$ compared with the $\mathrm{PA}+$ si-con group, ${ }^{\mathrm{c}} \mathrm{P}<0.05$ compared with the NC+si-NLRP3 group. Abbreviations: si-NLRP3, the podocytes transfected by the NLRP3-specific siRNA; si-con, the podocytes transfected by scrambled siRNA.

significantly decreased UACR and BUN levels compared to those in untreated DN rats (Figures 10 (a) and 10(b)). In addition, kidney tissues were collected for routine HE, PAS, and Masson staining at the end of the experiment. Furthermore, electron microscopy (EM) was used to observe the thickness of glomerular basement membrane (GBM). The results of HE, PAS, and Masson's staining demonstrated that the DN model rats presented obvious pathological changes compared with those of normal group, while Rg1 alleviated glomerular hypertrophy with diffuse and nodular sclerosis, excessive glycogen storage, and ultrastructural anomalies like glomerular podia cell fusion, rupture, and loss in the diabetic rats (Figure 10(c)). Taken together, Rg1 improved renal function and morphology in the DN rats. 


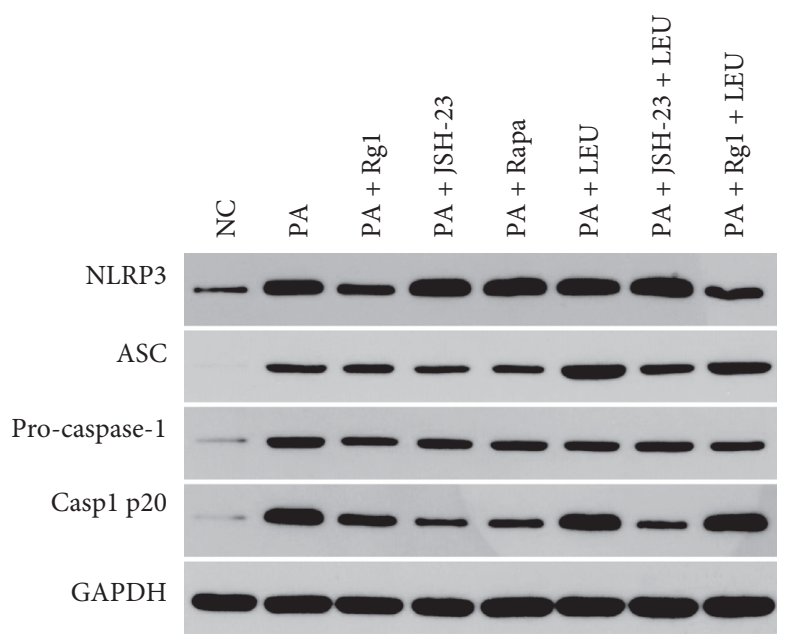

(a)

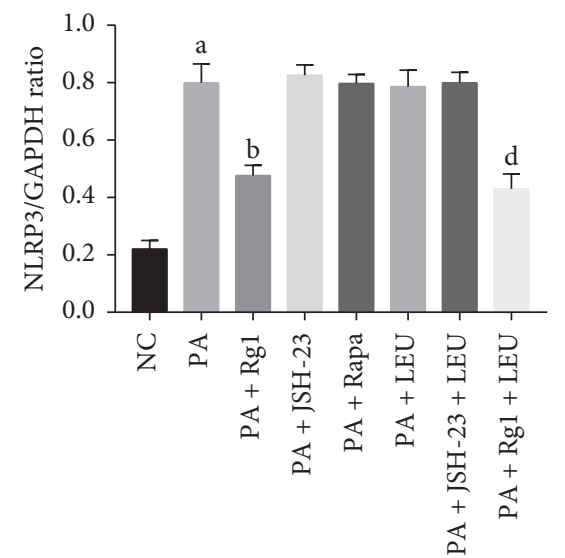

(b)

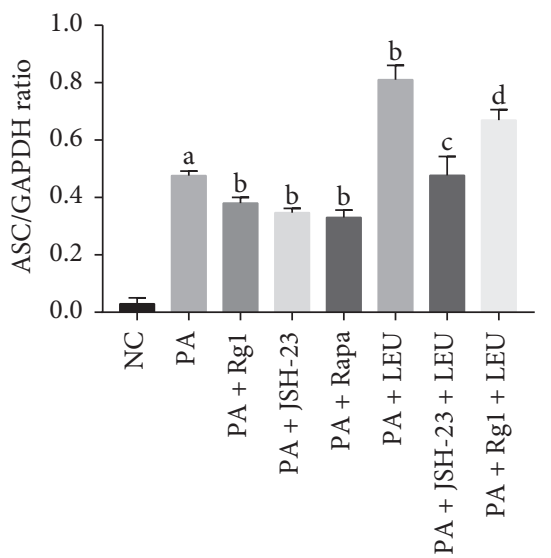

(c)

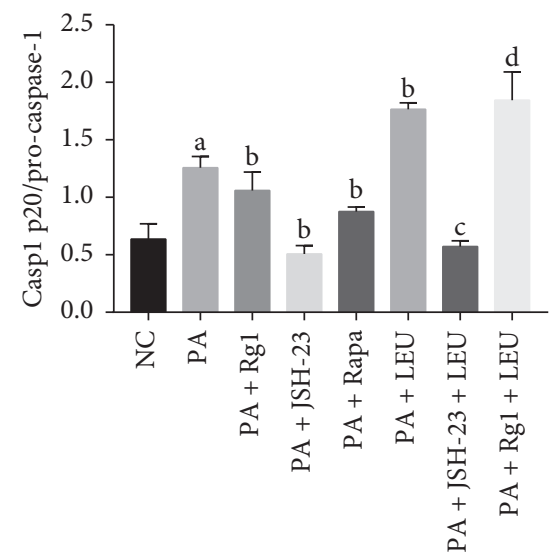

(d)

FIGURE 9: Rg1 inhibited the pyroptotic cascade in podocytes via the mTOR/NF- $\kappa$ B/NLRP3 axis: immunoblot and quantification of NLRP3, ASC, procaspase-1, and Casp1 p20 in podocytes treated as indicated. Values are the mean \pm SD; $n=3$. ${ }^{\text {a }} P<0.05$ compared with the NC group, ${ }^{b} P<0.05$ compared with the PA group, ${ }^{c} P<0.05$ compared with the $\mathrm{PA}+\mathrm{LEU}$ group, ${ }^{\mathrm{d}} \mathrm{P}<0.05$ compared with the $\mathrm{PA}+\mathrm{Rg} 1$ group.

\section{Discussion}

The ginsenoside $\mathrm{Rg} 1$ is one of the pharmacologically active components of ginseng and exhibits antiapoptotic, antiinflammatory, and neuroprotective effects [39-42]. It also has a significant reno-protective action [43] and alleviates aldosterone-induced podocyte injury [44]. In line with these findings, we found that $\mathrm{Rg} 1$ also protected podocytes from hyperlipidemic injury both in vivo and in vitro.

Pyroptosis is a form of programmed cell death mediated by the NLRP3 inflammasome and caspase-1 and is the pathological basis of various inflammatory and degenerative disorders $[17,35]$. It is frequently induced by hyperlipidemia and therefore associated with diabetic cardiomyopathy and diabetic retinopathy $[45,46]$. Recently, Wang et al. detected increased pyroptosis in the tubular cells involved in diabetic kidney disease (DKD) [7]. Similarly, we also observed pyroptosis in podocytes exposed to hyperlipidemic conditions, indicating its involvement in DN. The mTOR pathway has been previously implicated in podocyte injury [47] and was also upregulated in the affected cells in our study. In addition, there is a positive crosstalk between mTOR and $\mathrm{NF}-\kappa \mathrm{B}[48,49]$, and Wei et al. associated NF- $\kappa \mathrm{B}$ pathway activation with damaged podocytes [50]. Rg1 significantly inhibited pyroptosis, restored nephrin expression and cytoskeletal structure in the podocytes, and blocked hyperlipidemia-induced mTOR/NF- $\kappa \mathrm{B}$ signaling.

The pyroptotic cascade is triggered by the NLRP3 inflammasome, which then activates caspase- 1 and releases IL- $1 \beta$ [51-53]. While IL- $1 \beta$ mediates podocyte injury and renal inflammation [54], NLRP3 is involved in the pathogenesis of chronic kidney disease $[55,56]$. Since hyperlipidemia can activate multiple inflammasomes [57], we knocked down NLRP3 in podocytes and found that absence of NLRP3 inhibited pyroptosis, indicating that it initiates pyroptosis in podocytes during DN.

Since NF- $\kappa$ B activation is needed to activate the NLRP3 inflammasome [58-60], we also treated the podocytes with mTOR activator/inhibitor and NF- $\kappa$ B inhibitor. JSH23 and rapamycin both attenuated NLRP3 activation, while cells 


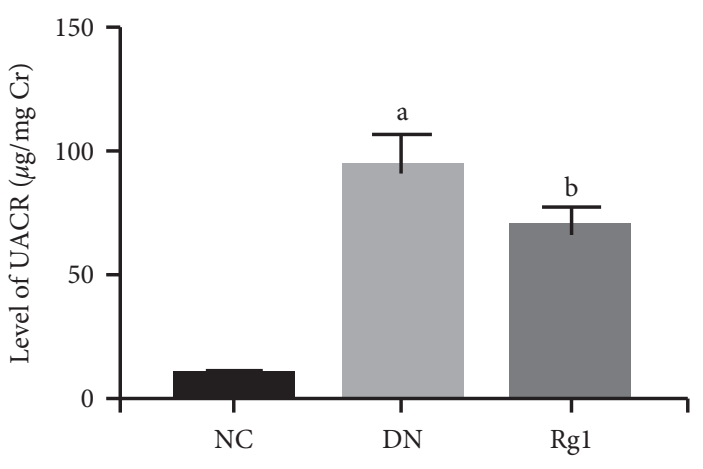

(a)

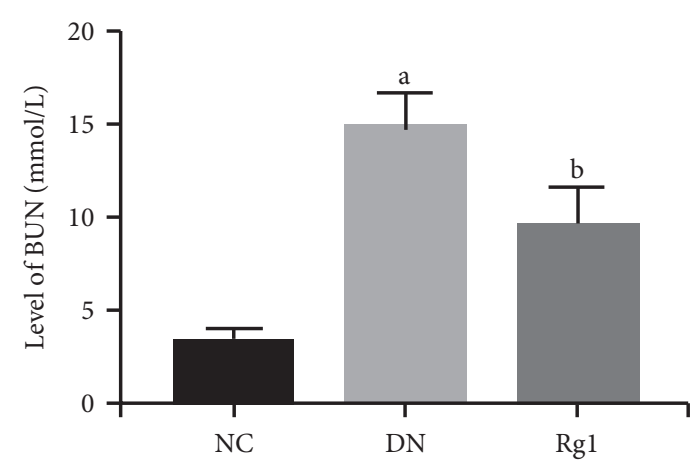

(b)
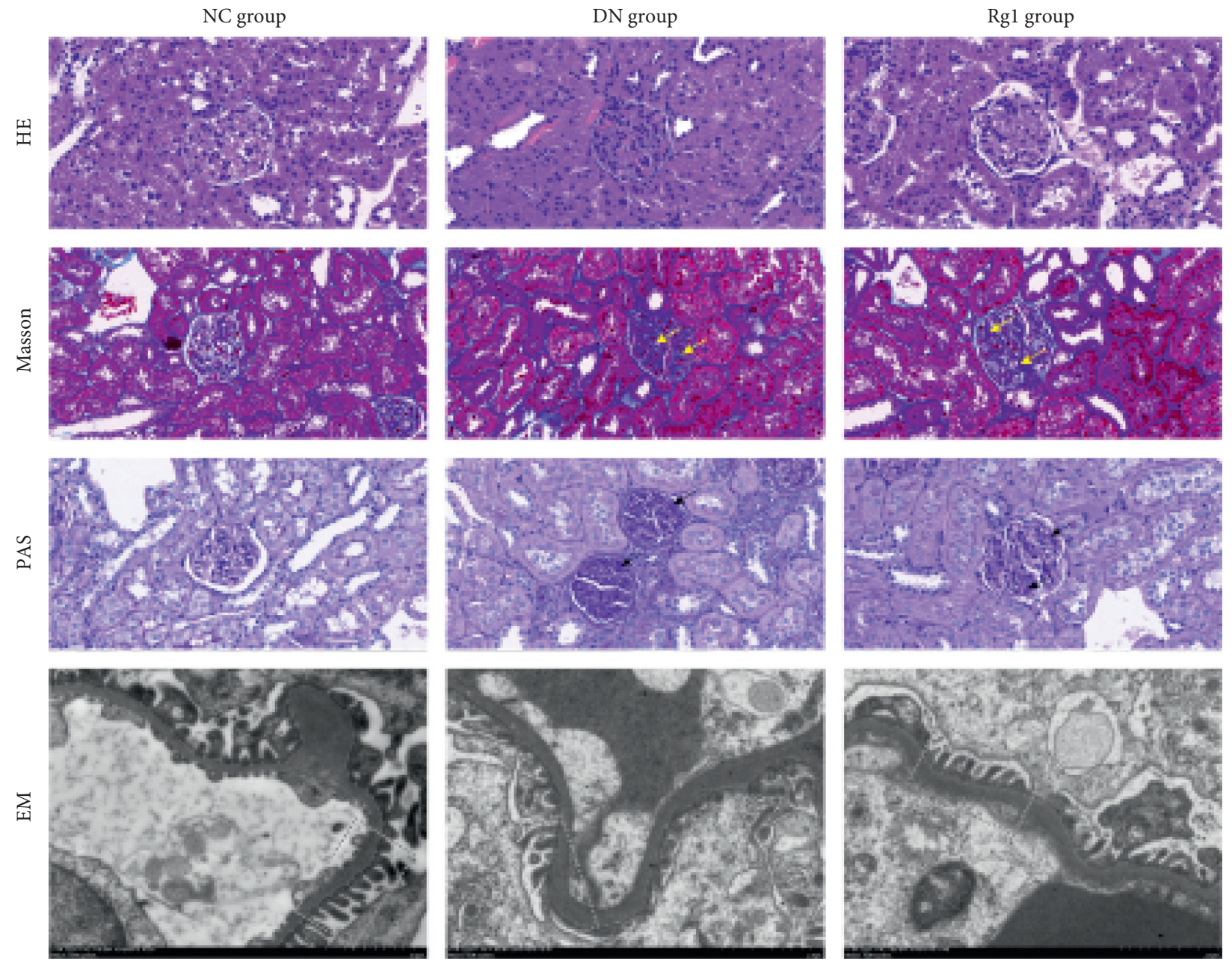

(c)

FIGURE 10: Rg1 improved renal function in DN rats. (a) and (b) UACR and BUN levels in the untreated and Rg1-treated DN rats. (c) Representative HE, PAS, Masson (original magnification 200x), and EM (original magnification 10,000x) images showing structure of the glomerular basement membrane (GBM) in the different groups. Values are the mean $\pm \mathrm{SD} ; n=3$. ${ }^{\mathrm{a}} P<0.05$ compared with the $\mathrm{NC}$ group, ${ }^{\mathrm{b}} P<0.05$ compared with the PA group. The yellow arrows show mesangial matrix. The black arrows show glycogen storage. The dotted yellow boxes indicate the GBM. Abbreviations: UACR, urine albumin creatinine ratio; BUN, blood urea nitrogen; HE, hematoxylin-eosin; PAS, periodic acid-Schiff; EM, electron microscope.

treated with LEU showed high levels of NLRP3, ASC, and Casp1 p20. In addition, JSH-23 abrogated the effect of LEU on the activation of NLRP3, indicating that mTOR activates NLRP3 via NF- $\kappa$ B. Rg1 also significantly decreased NLRP3 levels in the hyperlipidemia-exposed podocytes. Furthermore, the inhibitory effects of Rg1 on the activation of NLRP3 were reversed by LEU, which strongly suggested that Rg1 decreased hyperlipidemia-induced pyroptosis in 
podocytes by inhibiting the mTOR/NF- $\kappa \mathrm{B} / \mathrm{NLRP} 3$ axis. A previous study showed that the combination of $\mathrm{Rg} 1$ and astragaloside relieved renal fibrosis in $\mathrm{DN}$ by inhibiting the TGF- $\beta 1 /$ Smads pathway and oxidative stress [33]. Consistent with this, Rg1 significantly reduced proteinuria, improved renal function, and alleviated tissue damage in diabetic rats. Podocyte is the functional unit of the glomerular filtration membrane [61], and its injury is the pathological basis of DN [62]. Thus, Rg1-mediated podocyte rescue translated to improved renal function.

In summary, $\operatorname{Rg} 1$ improves renal function in $\mathrm{DN}$ rats by inhibiting hyperlipidemia-induced pyroptosis in podocytes via targeting $\mathrm{mTOR} / \mathrm{NF}-\kappa \mathrm{B} / \mathrm{NLRP} 3$ signaling. Our findings provide the experimental basis for future clinical trials on Rg1 as a treatment option for DN.

\section{Conclusions}

Ginsenoside $\operatorname{Rg} 1$ protects podocytes from hyperlipidemiainduced damage by inhibiting pyroptosis through the $\mathrm{mTOR} / \mathrm{NF}-\kappa \mathrm{B} / \mathrm{NLRP} 3$ axis, indicating a potential therapeutic function in $\mathrm{DN}$.

\section{Data Availability}

The data used to support the findings of this study are included within the article.

\section{Conflicts of Interest}

The authors declare no conflicts of interest.

\section{Acknowledgments}

The authors acknowledge the efforts and insights of their colleagues for this study. This study was supported by a grant from the Major National Basic Research Program of China 973 Program (no. 2012CB518602) and National Key R\&D Program of China (no. 2018YFC1704102).

\section{References}

[1] L. F. Fried, N. Emanuele, J. H. Zhang et al., "Combined angiotensin inhibition for the treatment of diabetic nephropathy," New England Journal of Medicine, vol. 369, no. 20, pp. 1892-1903, 2013.

[2] S. Hakroush, A. Cebulla, T. Schaldecker, D. Behr, P. Mundel, and A. Weins, "Extensive podocyte loss triggers a rapid parietal epithelial cell response," Journal of the American Society of Nephrology, vol. 25, no. 5, pp. 927-938, 2014.

[3] Z. S. Wang, F. Xiong, X. H. Xie, D. Chen, J. H. Pan, and L. Cheng, "Astragaloside IV attenuates proteinuria in streptozotocin-induced diabetic nephropathy via the inhibition of endoplasmic reticulum stress," BMC Nephrology, vol. 16, no. 1, p. 44, 2015.

[4] S. K. Mallipattu and J. C. He, "The podocyte as a direct target for treatment of glomerular disease?," American Journal of Physiology-Renal Physiology, vol. 311, no. 1, pp. F46-F51, 2016.

[5] M. Yasuda-Yamahara, S. Kume, A. Tagawa, H. Maegawa, and T. Uzu, "Emerging role of podocyte autophagy in the progression of diabetic nephropathy," Autophagy, vol. 11, no. 12, pp. 2385-2386, 2015.

[6] S. Ravindran, V. Kuruvilla, K. Wilbur, and S. Munusamy, "Nephroprotective effects of metformin in diabetic nephropathy," Journal of Cellular Physiology, vol. 232, no. 4, pp. 731-742, 2017.

[7] Y. Wang, X. Zhu, S. Yuan et al., “TLR4/NF- $\kappa$ B signaling induces GSDMD-related pyroptosis in tubular cells in diabetic kidney disease," Frontiers in Endocrinology, vol. 10, p. 603, 2019.

[8] Y. Liu, K. Lian, L. Zhang et al., "TXNIP mediates NLRP3 inflammasome activation in cardiac microvascular endothelial cells as a novel mechanism in myocardial ischemia/ reperfusion injury," Basic Research in Cardiology, vol. 109, no. 5, p. 415, 2014.

[9] M. Kitazawa, S. Hida, C. Fujii et al., "ASC induces apoptosis via activation of caspase- 9 by enhancing gap junction-mediated intercellular communication," PLoS One, vol. 12, no. 1, Article ID e0169340, 2018.

[10] E. A. Miao, I. A. Leaf, P. M. Treuting et al., "Caspase-1-induced pyroptosis is an innate immune effector mechanism against intracellular bacteria," Nature Immunology, vol. 11, no. 12, pp. 1136-1142, 2010.

[11] E. A. Miao, J. V. Rajan, and A. Aderem, "Caspase-1-induced pyroptotic cell death,” Immunological Reviews, vol. 243, no. 1, pp. 206-214, 2011.

[12] M.-S. Yoon, "The role of mammalian target of rapamycin (mTOR) in insulin signaling," Nutrients, vol. 9, no. 11, p. 1176, 2017.

[13] J. Cosin-Roger, S. Simmen, M. Hassan et al., "Hypoxia ameliorates intestinal inflammation through NLRP3/mTOR downregulation and autophagy activation," Nature Communications, vol. 8, no. 1, p. 98, 2017.

[14] X. Jia, Bo Liu, L. Bao et al., "Delayed oseltamivir plus sirolimus treatment attenuates H1N1 virus-induced severe lung injury correlated with repressed NLRP3 inflammasome activation and inflammatory cell infiltration," PLoS Pathogens, vol. 14, no. 11, Article ID e1007428, 2018.

[15] H. Wen, D. Gris, Y. Lei et al., "Fatty acid-induced NLRP3ASC inflammasome activation interferes with insulin signaling," Nature Immunology, vol. 12, no. 5, pp. 408-415, 2011.

[16] M. M. Hughes and L. A. J. O'Neill, "Metabolic regulation of NLRP3,” Immunological Reviews, vol. 281, no. 1, pp. 88-98, 2018.

[17] M. Li, X. E. Wang, M. Liu, X. Qi, and J. Li, "NF- $\kappa$ B signaling inhibition and anticancer activities of LLDT-246 on human colorectal cancer HCT-116 cells in vitro," Biomedicine \& Pharmacotherapy, vol. 68, no. 5, pp. 527-535, 2014.

[18] S. Sahan-Firat, M. Temiz-Resitogu, D. Sinem Guden et al., "Protection by mTOR inhibition on zymosan-induced systemic inflammatory response and oxidative/nitrosative stress: contribution of mTOR/MEK1/ERK1/2/IKK $\beta / \mathrm{I} \kappa \mathrm{B}-\alpha / \mathrm{NF}-\kappa \mathrm{B}$ signalling pathway," Inflammation, vol. 41, no. 1, pp. 272-298, 2018.

[19] V. Nikoletopoulou, M. Markaki, K. Palikaras, and N. Tavernarakis, "Crosstalk between apoptosis, necrosis and autophagy," Biochimica et Biophysica Acta (BBA)-Molecular Cell Research, vol. 1833, no. 12, pp. 3448-3459, 2013.

[20] M. Minhajuddin, F. Fazal, K. M. Bijli, M. R. Amin, and A. Rahman, "Inhibition of mammalian target of rapamycin potentiates thrombin-induced intercellular adhesion molecule- 1 expression by accelerating and stabilizing NF- $\kappa \mathrm{B}$ activation in endothelial cells," The Journal of Immunology, vol. 174, no. 9, pp. 5823-5829, 2005. 
[21] X. Wang, Y. Gao, N. Tian et al., “Astragaloside IV represses high glucose-induced mesangial cells activation by enhancing autophagy via SIRT1 deacetylation of NF- $\kappa$ B p65 subunit," Drug Design, Development and Therapy, vol. 12, pp. 29712980, 2018.

[22] S. Guan, W. Xu, F. Han et al., "Ginsenoside Rg1 attenuates cigarette smoke-induced pulmonary epithelial-mesenchymal transition via inhibition of the TGF- $\beta 1 /$ smad pathway," BioMed Research International, vol. 2017, Article ID 7171404, 12 pages, 2017.

[23] H.-T. Yu, J. Zhen, B. Pang, J.-N. Gu, and S.-S. Wu, "Ginsenoside Rg1 ameliorates oxidative stress and myocardial apoptosis in streptozotocin-induced diabetic rats," Journal of Zhejiang University-Science B, vol. 16, no. 5, pp. 344-354, 2015.

[24] Y. Li, F. Wang, and Y. Luo, "Ginsenoside Rg1 protects against sepsis-associated encephalopathy through beclin 1-independent autophagy in mice," Journal of Surgical Research, vol. 207, pp. 181-189, 2017.

[25] S. Bi, X. Ma, Y. Wang et al., "Protective effect of ginsenoside Rg1 on oxidative damage induced by hydrogen peroxide in chicken splenic lymphocytes," Oxidative Medicine and Cellular Longevity, vol. 2019, Article ID 8465030, 13 pages, 2019.

[26] L. N. Zhang, X. S. Xie, C. Zuo, and J. M. Fan, "Effect of ginsenoside Rgl on the expression of TNF-alpha and MCP-1 in rats with diabetic nephropathy," Sichuan Da Xue Xue Bao Yi Xue Ban, vol. 40, no. 3, pp. 466-471, 2009.

[27] P. Yang, L. Ling, W. Sun et al., "Ginsenoside Rg1 inhibits apoptosis by increasing autophagy via the AMPK/mTOR signaling in serum deprivation macrophages," Acta Biochimica et Biophysica Sinica, vol. 50, no. 2, pp. 144-155, 2018.

[28] T. Wang, Y. Gao, X. Wang et al., "Calpain-10 drives podocyte apoptosis and renal injury in diabetic nephropathy," Diabetes, Metabolic Syndrome and Obesity: Targets and Therapy, vol. 12, no. 12, pp. 1811-1820, 2019.

[29] X. Cai, L. Wang, X. Wang, and F. Hou, "miR-124a enhances therapeutic effects of bone marrow stromal cells transplant on diabetic nephropathy-related epithelial-to-mesenchymal transition and fibrosis," Journal of Cellular Biochemistry, vol. 121, no. 1, pp. 299-312, 2020.

[30] N. Tian, Y. Gao, X. Wang et al., "Emodin mitigates podocytes apoptosis induced by endoplasmic reticulum stress through the inhibition of the PERK pathway in diabetic nephropathy," Drug Design, Development and Therapy, vol. 12, no. 12, pp. 2195-2211, 2018.

[31] T. Hatta, S. I. Iemura, T. Ohishi et al., "Calpain-10 regulates actin dynamics by proteolysis of microtubule-associated protein 1B," Scientific Reports, vol. 8, no. 1, p. 16756, 2018.

[32] D. D. Arrington, T. R. Van Vleet, and R. G. Schnellmann, "Calpain 10: a mitochondrial calpain and its role in calciuminduced mitochondrial dysfunction," American Journal of Physiology-Cell Physiology, vol. 291, no. 6, pp. C1159-C1171, 2006.

[33] N. Du, Z. Xu, M. Gao, P. Liu, B. Sun, and X. Cao, "Combination of ginsenoside $\mathrm{Rg} 1$ and astragaloside IV reduces oxidative stress and inhibits TGF- $\beta 1 /$ smads signaling cascade on renal fibrosis in rats with diabetic nephropathy," Drug Design, Development and Therapy, vol. 12, pp. 3517-3524, 2018.

[34] M. Vitlov Uljević, K. Starčević, T. Mašek et al., "Dietary DHA/ EPA supplementation ameliorates diabetic nephropathy by protecting from distal tubular cell damage," Cell and Tissue Research, vol. 378, no. 2, pp. 301-307, 2019.
[35] S. M. Man, R. Karki, T.-D. Kanneganti, and K. ThirumalaDevi, "Molecular mechanisms and functions of pyroptosis, inflammatory caspases and inflammasomes in infectious diseases," Immunological Reviews, vol. 277, no. 1, pp. 61-75, 2017.

[36] Yu Liu, J. Zhang, Y. Wang, and X. Zeng, "Apelin involved in progression of diabetic nephropathy by inhibiting autophagy in podocytes," Cell Death \& Disease, vol. 8, no. 8, p. e3006, 2017.

[37] J. Jin, K. Hu, M. Ye, D. Wu, and Q. He, "Rapamycin reduces podocyte apoptosis and is involved in autophagy and mTOR/ P70S6K/4EBP1 signaling," Cellular Physiology and Biochemistry, vol. 48, no. 2, pp. 765-772, 2018.

[38] X. Li, X. Zhang, Y. Pan et al., "mTOR regulates NLRP3 inflammasome activation via reactive oxygen species in murine lupus," Acta Biochimica et Biophysica Sinica, vol. 50, no. 9, pp. 888-896, 2018.

[39] G. Zu, J. Guo, N. Che, T. Zhou, and X. Zhang, "Protective effects of ginsenoside $\operatorname{Rg} 1$ on intestinal ischemia/reperfusion injury-induced oxidative stress and apoptosis via activation of the Wnt/beta-catenin pathway," Scientific Reports, vol. 6, p. 38480, 2016.

[40] H. Yu, J. Zhen, Y. Yang, J. Gu, S. Wu, and Q. Liu, "Ginsenoside Rg1 ameliorates diabetic cardiomyopathy by inhibiting endoplasmic reticulum stress-induced apoptosis in a streptozotocin-induced diabetes rat model," Journal of Cellular and Molecular Medicine, vol. 20, no. 4, pp. 623-631, 2016.

[41] J. Li, C. Yang, S. Zhang et al., "Ginsenoside Rg1 inhibits inflammatory responses via modulation of the nuclear factor$\kappa \mathrm{B}$ pathway and inhibition of inflammasome activation in alcoholic hepatitis," International Journal of Molecular Medicine, vol. 41, no. 2, pp. 899-907, 2018.

[42] Y. B. Li, Y. Wang, J. P. Tang, D Chen, and S. L Wang, "Neuroprotective effects of ginsenoside Rg1-induced neural stem cell transplantation on hypoxic-ischemic encephalopathy," Neural Regeneration Research, vol. 10, no. 5, pp. 753-759, 2015.

[43] T. Yokozawa and E. Dong, "Role of ginsenoside-Rd in cisplatin-induced renal injury: special reference to DNA fragmentation," Nephron, vol. 89, no. 4, pp. 433-438, 2001.

[44] N. Mao, Y. Cheng, X.-L. Shi et al., "Ginsenoside Rg1 protects mouse podocytes from aldosterone-induced injury in vitro," Acta Pharmacologica Sinica, vol. 35, no. 4, pp. 513-522, 2014.

[45] R.-C. Yue, S.-Z. Lu, Yu Luo et al., "Calpain silencing alleviates myocardial ischemia-reperfusion injury through the NLRP3/ ASC/caspase-1 axis in mice," Life Sciences, vol. 233, Article ID 116631, 2019.

[46] C. Gu, D. Draga, C. Zhou et al., "miR-590-3p inhibits pyroptosis in diabetic retinopathy by targeting NLRP1 and inactivating the NOX4 signaling pathway," Investigative Opthalmology \& Visual Science, vol. 60, no. 13, pp. 4215-4223, 2019.

[47] K. Inoki, H. Mori, J. Wang et al., "mTORC1 activation in podocytes is a critical step in the development of diabetic nephropathy in mice," Journal of Clinical Investigation, vol. 121, no. 6, pp. 2181-2196, 2011.

[48] X. Yu, D. Jin, A. Yu, J. Sun, X. Chen, and Z. Yang, "p65 downregulates DEPTOR expression in response to LPS stimulation in hepatocytes," Gene, vol. 589, no. 1, pp. 12-19, 2016.

[49] A. Criollo, F. Chereau, S. A. Malik et al., "Autophagy is required for the activation of $\mathrm{NF} \kappa \mathrm{B}$," Cell Cycle, vol. 11, no. 1, pp. 194-199, 2012.

[50] M. Wei, Z. Li, and Z. Yang, "Crosstalk between protective autophagy and NF- $\kappa \mathrm{B}$ signal in high glucose-induced 
podocytes," Molecular and Cellular Biochemistry, vol. 394, no. 1-2, pp. 261-273, 2014.

[51] S. Mariathasan, D. S. Weiss, K. Newton et al., "Cryopyrin activates the inflammasome in response to toxins and ATP," Nature, vol. 440, no. 7081, pp. 228-232, 2006.

[52] T. D. Kanneganti, M. Body Malapel, A. Amer et al., "Critical role for cryopyrin/ $/ \mathrm{Nalp}_{3}$ in activation of caspase-1 in response to viral infection and double-stranded RNA," The Journal of Biological Chemistry, vol. 281, no. 48, pp. 36560-36568, 2006.

[53] C. Zhang, K. M. Boini, M. Xia et al., "Activation of nod-like receptor protein 3 inflammasomes turns on podocyte injury and glomerular sclerosis in hyperhomocysteinemia," Hypertension, vol. 60, no. 1, pp. 154-162, 2012.

[54] Z. I. Niemir, H. Stein, G. Dworacki et al., "Podocytes are the major source of IL- $1 \alpha$ and IL- $1 \beta$ in human glomerulonephritides," Kidney International, vol. 52, no. 2, pp. 393-403, 1997.

[55] S. Iyer, W. P. Pulskens, J. Sadler et al., "Necrotic cells trigger a sterile inflammatory response through the NLRP3 inflammsome," Proceedings of the National Academy of Sciences, vol. 106, no. 48, pp. 20388-20393, 2009.

[56] K. Schroder, R. Zhou, and J. Tschopp, "The NLRP3 inflammasome: a sensor for metabolic danger?," Science, vol. 327, no. 5963, pp. 296-300, 2010.

[57] S. Corrêa-Silva, A. P. Alencar, J. B. Moreli et al., "Hyperglycemia induces inflammatory mediators in the human chorionic villous," Cytokine, vol. 111, pp. 41-48, 2018.

[58] H. Yi, R. Peng, L.-Y. Zhang et al., "LincRNA-Gm4419 knockdown ameliorates NF- $\kappa \mathrm{B} / \mathrm{NLRP} 3$ inflammasome-mediated inflammation in diabetic nephropathy," Cell Death \& Disease, vol. 8, no. 2, p. e2583, 2017.

[59] W.-J. Su, Y. Zhang, Y. Chen et al., "NLRP3 gene knockout blocks NF- $\kappa$ B and MAPK signaling pathway in CUMS-induced depression mouse model," Behavioural Brain Research, vol. 322, pp. 1-8, 2017.

[60] X. Yu, P. Lan, X. Hou et al., "HBV inhibits LPS-induced NLRP3 inflammasome activation and IL- $1 \beta$ production via suppressing the NF- $\kappa \mathrm{B}$ pathway and ROS production," Journal of Hepatology, vol. 66, no. 4, pp. 693-702, 2017.

[61] E. Torban, F. Braun, N. Wanner et al., "From podocyte biology to novel cures for glomerular disease," Kidney International, vol. 96, no. 4, pp. 850-861, 2019.

[62] A. A. Eid, Y. Gorin, B. M. Fagg et al., "Mechanisms of podocyte injury in diabetes: role of cytochrome $\mathrm{P} 450$ and NADPH oxidases," Diabetes, vol. 58, no. 5, pp. 1201-1211, 2009. 